\title{
Seasonal Diversity of Birds and Ecosystem Services in Agricultural Area of Western Ghats, Maharashtra State, India
}

\author{
M.R.Abdar \\ Dept. of zoology Krantisinh Nana Patil College Walwe Dist.Sangli (M.S.) India
}

\begin{abstract}
Seasonal diversity of birds and ecosystem services in agricultural area was studied in 2009-2012 by using line transect method. Maximum bird's species avoid agricultural area nearly one third of all observed birds regularly to occasionally use such habitat, often providing important ecosystem services like pest control, pollination and seed disposal. I compared bird's species that prefer agricultural area with respect to diet, habitat, rang and population size and resources specialization. The species richness and diversity was more in winter, especially Insectivores, frugivores, granivores and omnivores. In contrast, species richness and diversity less in rainy season. These finding indicate that winter is favorable for breeding, feeding and foraging for many omnivores, granivores, frugivores than other two season even agricultural area providing important ecosystem services.
\end{abstract}

Key words: Agricultural area, birds, diversity, Ecosystem services, Western Gjhats.

\section{Introduction}

The bird habitats of the Indian subcontinent can be roughly divided into forest, scrub, wetlands, grassland, desert and agricultural land. The forest areas of the region are vitally important for many birds. Relatively few birds are characteristics of scrub mixed with grassland. Wetlands are abundant in the region and support rich array of waterfowl. They are major staging and wintering ground for waterfowl. Agriculture birds constitute an important component. The dual role of bird in agriculture is very well known (Ali 1971).Agriculture provides a concentrated and highly predictable source of food to bird. This food includes grains, seeds, fruits, green vegetation of crop plants, Insects, rodents and arthropods ('O'Cannor and Shrub 1986).Agricultural land scapes in Maharashtra, especially in the intensively cultivated area like South Maharashtra have number of dairy and poultry farms interspersed among crop field. These farms along with a variety of agroforstry trees provide additional food to birds in the form of animal feeds, tree- fruits, seeds, nectar etc.Birds of agricultural area therefore include granivores, frugivores, insectivores and omnivores. Although less than $1 \%$ of the world's birds species primarily prefer agricultural area. Nearly a third of all birds species occasionally use such habitats (Sekercioglu et al.,2007), often providing important ecosystem services, such as pest control, pollination, seed dispersal (Sekercioglu 2006). Agriculture ornithology helps us to obtain scientific information on birds in relation to agriculture.This information useful to their management and conservation of useful species of birds and control of pest birds.There is growing interest in avian diversity in agricultural area such as Shindsa and Saini 1994, Wang et al.,2003,Naidoo R. 2004,Waltert et al., 2005,Perfecto et al., 2008,Tscharntke et al., 2008, Van Bael et al., 2008, Clough et al., 2011, Sekercioglu 2012.

The objective of this study is

$>\quad$ To obtain scientific information on birds, whether it is useful or harmful for agriculture.

$>\quad$ To study in detail the food and feeding behavior.

$>\quad$ To study in their reproduction, roosting and population dynamics.

\section{Material and Methods}

Some of the basic methods used in this study as described by Bibby et al. (1992) are: Point counts: to determine abundance by undertaking a bird count from a fixed location for fixed period of time. The bird species seen or heard are recorded.

Line transect: suitable for estimating density and abundance which involves moving along a fixed route ( transect) and recording the bird species seen and heard on both side of the transect.

The study was conducted in between September 2009 to August 2012. The bird counts were carried out in the morning between 7.00 am to $10.00 \mathrm{am}$.and evening between $4.00 \mathrm{pm}$ to $6.00 \mathrm{pm} 10$ by 40 Olympus binocular was used to confirm the identification of the birds, nests located by sight. Data analysis:

a. Species composition: abundance for each species was calculated by summing up the number of individuals recorded in all the transect.

b. $\quad$ Species diversity using Simpson index (D), Simpson Diversity index ( 1-D) and Simpsons Reciprocal index $(1 / \mathrm{D})$ 


\section{Result and Discussion}

Table 1. Birds observed in agricultural area of Western Ghats, Maharashtra 2009-2012

\begin{tabular}{|c|c|}
\hline 1. & Intermediate Egret Mesophoyx intermedia \\
\hline 2. & Little Egret Egretta garzatta \\
\hline 3. & Cattle Egret Bubulcus ibis \\
\hline 4. & Little Heron Butorides striatus \\
\hline 5. & Darter Anbinga melanogaster \\
\hline 6. & Indian Cormorant Phalacrocorax fuscicollis \\
\hline 7. & Grey Heron Ardea cinerea \\
\hline 8. & Woolly-necked Stork Ciconia episcopus \\
\hline 9. & Black Ibis Pseudibis papillosa \\
\hline 10. & Black- shouldered Kite Elanus caeruleus \\
\hline 11. & Black Kite Milvus migrans \\
\hline 12. & Shikra Accipiter badius \\
\hline 13. & Pallid Harrier Circus macrourus \\
\hline 14. & Jungle Bush Quail Perdicula asiatica \\
\hline 15. & Yellow-legged Buttonquail Turnix tanki \\
\hline 16. & Indian Peafowl Pavo cristatus \\
\hline 17. & Demoiselle Crane Grus virgo \\
\hline 18. & White-breasted Waterhen Amaurornis phoenicurus \\
\hline 19. & Purple Swamphen Porphyrio porphyrio \\
\hline 20. & Black-winged Stilt Himantopus bimantopus \\
\hline 21. & Indian Courser Cursorius coromandelicus \\
\hline 22. & Red-wattled Lapwing Vanellus indicus \\
\hline 23. & Common Sandpiper Actitis hypoleucos \\
\hline 24. & Little Stint Calidris minuta \\
\hline 25. & Little Ringed Plover Charadrius dubius \\
\hline 26. & River Tern Sterna aurantia \\
\hline 27. & Eurasian Collared Dove Streptopelia decaocto \\
\hline 28. & Spotted Dove Streptopelia chinensis \\
\hline 29. & Rock Pigeon Columba livia \\
\hline 30. & Rose-ringed Parakeet Psittacula krameri \\
\hline 31. & Common Hawk Cuckoo Hierococcyx varius \\
\hline 32. & Asian Koel Eudynamys scolopacea \\
\hline 33. & Greater Coucal Centropus sinensis \\
\hline 34. & Barn Owl Tyto alba \\
\hline 35. & Asian Palm Swift Cypsiurus balasiensis \\
\hline 36. & Common Swift Apus apus \\
\hline 37. & House Swift Apus affinis \\
\hline 38. & Indian Roller Coracias bengbalensis \\
\hline 39. & Pied Kingfisher Ceryle rudis \\
\hline 40. & Stork-billed Kingfisher Halcyon pileata \\
\hline 41. & White-throated Kingfisher Halcyon smyrnensis \\
\hline 42. & Common Kingfisher Alcedo attbis \\
\hline 43. & Green Bee-eater Merops orientalis \\
\hline 44. & Chestnut-headed Bee-eater Merops leschenaulti \\
\hline 45. & Coppersmith Barbet Megalaima baemacephala \\
\hline 46. & Common Hoopoe Upupa epops \\
\hline 47. & Indian Grey Hornbill Ocyceros birostris \\
\hline 48. & Streak-throated Woodpecker Picus xanthopygaeus \\
\hline 49. & Yellow-crowned Woodpecker Dendrocopos mabrattensis \\
\hline 50. & Common Iora Aegithina tipbia \\
\hline 51. & Indian Bushlark Mirafra erythroptera \\
\hline 52. & Ashy-crowned Sparrow Lark Eremopterix grisea \\
\hline 53. & Oriental Skylark Alauda gulgula \\
\hline 54. & Crested Lark Galerida deva \\
\hline 55. & Wire-tailed Swallow Hirundo rustica \\
\hline 56. & Streak-throated Swallow Hirundo fluvicola \\
\hline 57. & Black Drongo Dicrurus macrocercus \\
\hline
\end{tabular}


58. Greater Racket-tailed Drongo Dicrurus paradiseus

59. White-billied Drongo Dicrurus caerulescens

60. Common Woodshrike Tephrodornis pondicerianus

61. Long-tailed Shrike Lanius schach

62. Bay-backed Shrike Lanius vittatus

63. Rosy Starling Sturnus roseus

64. Common Myna Acridotheres tristis

65. Jungle Myna Acridotheres fuscus

66. House Crow Corvus splendens

67. Large-billed Crow Corvus macrohynchos

68. Small Minivet Pericrocotus cinnamomeus

69. Red-vented Bulbul Pycnonotus cafer

70. Yellow-eyed Babbler Chrysomma sinense

71. Jungle Babbler Turdoides striatus

72. Large Grey Babbler Turdoides malcolmi

73. Common Babbler Turdoides caudatus

74. White-throated Fantail Rhipidura albicollis

75. Grey-breasted Prinia Prinia hodgsonii

76. Ashy Prinia Prinia socialis

77. Plain Prinia Prinia inornata

78. Jungle Prinia Prinia sylvatica

79. Common Tailorbird Orthotomus sutorius

80. Greenish Warbler Phylloscopus trochiloides

81. White-rumped Shama Copsycbus malabaricus

82. Oriental Magpie Robin Copsycbus saularis

83. Indian Robin Saxicoloides fulicata

84. Common Stonechat Saxicola torquata

85. Great Tit Parus major

86. Paddyfield Pipit Anthus rufulus

87. White-browed Wagtail Motacilla maderaspatensis

88. Grey Wagtail Motacilla cinerea

89. White Wagtail Motacilla alba

90. Yellow Wagtail Motacilla flava

91. Oriental White-eye Zosterops palpebrosus

92. Purple-rumped Sunbird Nectarinia zeylonica

93. Purple Sunbird Nectarinia asiatica

94. Indian Silverbill Lonchura malabarica

95. Scaly-breasted Munia Lonchura punctulata

96. House Sparrow Passer domesticus

97. Baya Weaver Ploceus philippinus

98. Crested Bunting Melophus lathami

Table 2. Birds in different habitats of Western Ghats, Maharashtra 2009-2012

\begin{tabular}{|l|l|l|l|l|}
\hline Sr.No & \multicolumn{1}{|c|}{ Name of Bird } & Worest habitat & Wetland habitat & Agricultural habitat \\
\hline 1 & Intermediate Egret & - & $\sqrt{ }$ & - \\
\hline 2 & Little Egret & -- & $\sqrt{ }$ & - \\
\hline 3 & Cattle Egret & - & $\sqrt{ }$ & - \\
\hline 4 & Little Heron & - & $\sqrt{ }$ & - \\
\hline 5 & Darter & - & $\sqrt{ }$ & - \\
\hline 6 & Indian Cormorant & - & $\sqrt{ }$ & - \\
\hline 7 & Grey Heron & - & $\sqrt{ }$ & - \\
\hline 8 & Wooly-necked Stork & - & $\sqrt{ }$ & - \\
\hline 9 & Black Ibis & - & $\sqrt{ }$ & - \\
\hline 10 & Black-shouldered Kite & $\sqrt{ }$ & - & - \\
\hline 11 & Black Kite & $\sqrt{ }$ & - & - \\
\hline 12 & Shikra & $\sqrt{ }$ & - & - \\
\hline 13 & Pallid Harrier & $\sqrt{ }$ & - & - \\
\hline 14 & Jungle Bush Quill & $\sqrt{ }$ & - & $\sqrt{ }$ \\
\hline 15 & Yellow -legged Buttonquail & - & - & \\
\hline 16 & Indian Peafowl & - & - & - \\
\hline 17 & Demoiselle Crane & - & - & $\sqrt{ }$ \\
\hline 18 & White-breasted Waterhen & - & $\sqrt{ }$ & - \\
\hline
\end{tabular}


Seasonal Diversity Of Birds And Ecosystem Services In Agricultural Area Of Western Ghats,

\begin{tabular}{|c|c|c|c|c|}
\hline 19 & Purple Swamphen & - & $\sqrt{ }$ & - \\
\hline 20 & Black-winged Stilt & - & $\sqrt{ }$ & - \\
\hline 21 & Indian Courser & - & $\sqrt{ }$ & $\sqrt{ }$ \\
\hline 22 & Red-wattled Lapwing & - & $\sqrt{ }$ & $\sqrt{ }$ \\
\hline 23 & Common Sandpiper & - & $\sqrt{ }$ & - \\
\hline 24 & Little Stint & - & $\sqrt{ }$ & - \\
\hline 25 & Little Ringed Plover & - & $\sqrt{ }$ & - \\
\hline 26 & River Tern & - & $\sqrt{ }$ & - \\
\hline 27 & Eurasian Collared Dove & - & - & $\sqrt{ }$ \\
\hline 28 & Spotted Dove & - & - & $\sqrt{ }$ \\
\hline 29 & Rock Pigeon & - & - & $\sqrt{ }$ \\
\hline 30 & Rose-ringed Parakeet & - & - & $\sqrt{ }$ \\
\hline 31 & Common Hawk Cuckoo & - & - & $\sqrt{ }$ \\
\hline 32 & Asian Koel & - & - & $\sqrt{ }$ \\
\hline 33 & Greater Coucal & - & - & $\sqrt{ }$ \\
\hline 34 & Barn Owl & $\sqrt{ }$ & - & - \\
\hline 35 & Asian Palm Swift & - & - & $\sqrt{ }$ \\
\hline 36 & Common Swift & - & - & $\sqrt{ }$ \\
\hline 37 & House Swift & - & - & $\sqrt{ }$ \\
\hline 38 & Indian Roller & - & - & $\sqrt{ }$ \\
\hline 39 & Pied Kingfisher & - & $\sqrt{ }$ & - \\
\hline 40 & Stork-billed Kingfisher & - & $\sqrt{ }$ & - \\
\hline 41 & White-throated Kingfisher & - & $\sqrt{ }$ & - \\
\hline 42 & Common Kingfisher & - & $\sqrt{ }$ & $\sqrt{ }$ \\
\hline 43 & Green Bee-eater & - & - & $\sqrt{ }$ \\
\hline 44 & Chestnut-headed Bee-eater & - & - & $\sqrt{ }$ \\
\hline 45 & Coppersmith Barbet & - & - & $\sqrt{ }$ \\
\hline 46 & Common Hoopoe & - & - & $\sqrt{ }$ \\
\hline 47 & Indian Grey Hornbill & $\sqrt{ }$ & - & $\sqrt{ }$ \\
\hline 48 & Streak-throated Woodpecker & $\sqrt{ }$ & - & $\sqrt{ }$ \\
\hline 49 & Yellow-crowned Woodpecker & $\sqrt{ }$ & - & - \\
\hline 50 & Common Iora & $\sqrt{ }$ & - & $\sqrt{ }$ \\
\hline 51 & Indian Bushlark & $\sqrt{ }$ & - & - \\
\hline 52 & Ashy-crowned Sparrow Lark & - & - & $\sqrt{ }$ \\
\hline 53 & Oriental Skylark & - & - & $\sqrt{ }$ \\
\hline 54 & Crested Lark & - & - & $\sqrt{ }$ \\
\hline 55 & Wire-tailed Swallow & - & $\sqrt{ }$ & $\sqrt{ }$ \\
\hline 56 & Streak-throated Swallow & - & $\sqrt{ }$ & $\sqrt{ }$ \\
\hline 57 & Black Drongo & - & - & $\sqrt{ }$ \\
\hline 58 & Greater Racket-tailed Drongo & - & - & $\sqrt{ }$ \\
\hline 59 & White-billed Drongo & - & - & $\sqrt{ }$ \\
\hline 60 & Common Woodshrike & - & - & $\sqrt{ }$ \\
\hline 61 & Long-tailed Shrike & - & - & $\sqrt{ }$ \\
\hline 62 & Rosy Starling & - & - & $\sqrt{ }$ \\
\hline 63 & Common Myna & $\sqrt{ }$ & - & $\sqrt{ }$ \\
\hline 64 & Jungle Myna & - & - & $\sqrt{ }$ \\
\hline 65 & House Crow & - & - & $\sqrt{ }$ \\
\hline 66 & Large-billed Crow & $\sqrt{ }$ & - & $\sqrt{ }$ \\
\hline 67 & Small Minivet & - & - & $\sqrt{ }$ \\
\hline 68 & Red-vented Bulbul & - & - & $\sqrt{ }$ \\
\hline 69 & Yellow-eyed Babbler & - & - & $\sqrt{ }$ \\
\hline 70 & Jungle Babbler & $\sqrt{ }$ & - & $\sqrt{ }$ \\
\hline 71 & Large Grey Babbler & - & - & $\sqrt{ }$ \\
\hline 72 & Common Babbler & - & - & $\sqrt{ }$ \\
\hline 73 & White-throated Fantail & - & - & $\sqrt{ }$ \\
\hline 74 & Grey-breasted Prinia & $\sqrt{ }$ & - & $\sqrt{ }$ \\
\hline 75 & Ashy Prinia & $\sqrt{ }$ & - & $\sqrt{ }$ \\
\hline 76 & Plain Prinia & $\sqrt{ }$ & - & $\sqrt{ }$ \\
\hline 77 & Jungle Prinia & $\sqrt{ }$ & - & $\sqrt{ }$ \\
\hline 78 & Common Tailorbird & - & - & $\sqrt{ }$ \\
\hline 79 & Greenish Warbler & - & - & $\sqrt{ }$ \\
\hline 80 & White-rumped Shama & - & - & $\sqrt{ }$ \\
\hline 81 & Oriental Magpie Robin & - & - & $\sqrt{ }$ \\
\hline 82 & Indian Robin & - & - & $\sqrt{ }$ \\
\hline 83 & Common Stonechat & - & - & $\sqrt{ }$ \\
\hline 84 & Great Tit & - & - & $\sqrt{ }$ \\
\hline 85 & Paddyfield Pipit & - & - & $\sqrt{ }$ \\
\hline 86 & White-browed Wagtail & - & $\sqrt{ }$ & - \\
\hline 87 & Grey Wagtail & - & $\sqrt{ }$ & $\sqrt{ }$ \\
\hline 88 & White Wagtail & - & $\sqrt{ }$ & $\sqrt{ }$ \\
\hline
\end{tabular}


Seasonal Diversity Of Birds And Ecosystem Services In Agricultural Area Of Western Ghats,

\begin{tabular}{|l|l|l|l|l|}
\hline 89 & Yellow Wagtail & - & $\sqrt{ }$ & $\sqrt{ }$ \\
\hline 90 & Oriental White-eye & - & - & $\sqrt{ }$ \\
\hline 91 & Purple-rumped Sunbird & - & - & $\sqrt{ }$ \\
\hline 92 & Purple Sunbird & - & - & $\sqrt{ }$ \\
\hline 93 & Indian Silverbill & - & - & $\sqrt{ }$ \\
\hline 94 & Scaly-breasted Munia & - & - & $\sqrt{ }$ \\
\hline 95 & House Sparrow & - & - & $\sqrt{ }$ \\
\hline 96 & Baya Weaver & - & - & \\
\hline 97 & Crested Bunting & $\sqrt{ }$ & - & - \\
\hline
\end{tabular}

Table 3. Seasonal diversity of Birds in Agricultural Area of Western Ghats, Maharashtra 2009-2012

\begin{tabular}{|c|c|c|c|c|}
\hline Sr.No & Name of Bird & Winter Season & Summer Season & Rainy Season \\
\hline 1 & Yellow -legged Buttonquail & 25 & 10 & 3 \\
\hline 2 & Indian Peafowl & 5 & 5 & - \\
\hline 3 & Demoiselle Crane & 25 & - & - \\
\hline 4 & Eurasian Collared Dove & 10 & 22 & - \\
\hline 5 & Spotted Dove & 11 & 10 & 8 \\
\hline 6 & Rock Pigeon & 15 & - & - \\
\hline 7 & Rose-ringed Parakeet & 20 & 7 & - \\
\hline 8 & Common Hawk Cuckoo & 6 & 4 & - \\
\hline 9 & Asian Koel & 8 & 5 & - \\
\hline 10 & Greater Coucal & 5 & 3 & 3 \\
\hline 11 & Asian Palm Swift & - & 10 & - \\
\hline 12 & Common Swift & - & 12 & 5 \\
\hline 13 & House Swift & - & 11 & - \\
\hline 14 & Indian Roller & 8 & - & - \\
\hline 15 & Green Bee-eater & - & 5 & 10 \\
\hline 16 & Chestnut-headed Bee-eater & - & - & 5 \\
\hline 17 & Coppersmith Barbet & 3 & - & 10 \\
\hline 18 & Common Hoopoe & - & - & 6 \\
\hline 19 & Ashy-crowned Sparrow Lark & - & 10 & - \\
\hline 20 & Oriental Skylark & - & 15 & - \\
\hline 21 & Crested Lark & - & 15 & - \\
\hline 22 & Black Drongo & 9 & 7 & - \\
\hline 23 & Greater Racket-tailed Drongo & 10 & - & - \\
\hline 24 & White-billed Drongo & 9 & - & - \\
\hline 25 & Common Woodshrike & 10 & - & - \\
\hline 26 & Long-tailed Shrike & 9 & - & - \\
\hline 27 & Rosy Starling & 500 & - & - \\
\hline 28 & Jungle Myna & - & 16 & - \\
\hline 29 & House Crow & 10 & 9 & 4 \\
\hline 30 & Small Minivet & 9 & - & 14 \\
\hline 31 & Red-vented Bulbul & 10 & 10 & 4 \\
\hline 32 & Yellow-eyed Babbler & - & 6 & - \\
\hline 33 & Large Grey Babbler & 23 & 10 & 4 \\
\hline 34 & Common Babbler & - & 10 & - \\
\hline 35 & White-throated Fantail & 22 & 4 & 2 \\
\hline 36 & Common Tailorbird & 23 & 5 & - \\
\hline 37 & Greenish Warbler & 12 & 10 & 4 \\
\hline 38 & White-rumped Shama & 8 & 10 & 3 \\
\hline 39 & Oriental Magpie Robin & 5 & 3 & - \\
\hline 40 & Indian Robin & 2 & 2 & 2 \\
\hline 41 & Common Stonechat & 12 & 4 & 3 \\
\hline 42 & Great Tit & 10 & 7 & - \\
\hline 43 & Paddyfield Pipit & 2 & - & 7 \\
\hline 44 & Oriental White-eye & - & - & 6 \\
\hline 45 & Purple-rumped Sunbird & 9 & 5 & 2 \\
\hline 46 & Purple Sunbird & 5 & 3 & - \\
\hline 47 & Indian Silverbill & - & - & 14 \\
\hline 48 & Scaly-breasted Munia & - & - & 12 \\
\hline 49 & House Sparrow & 15 & 20 & 8 \\
\hline 50 & Baya Weaver & 300 & - & - \\
\hline
\end{tabular}




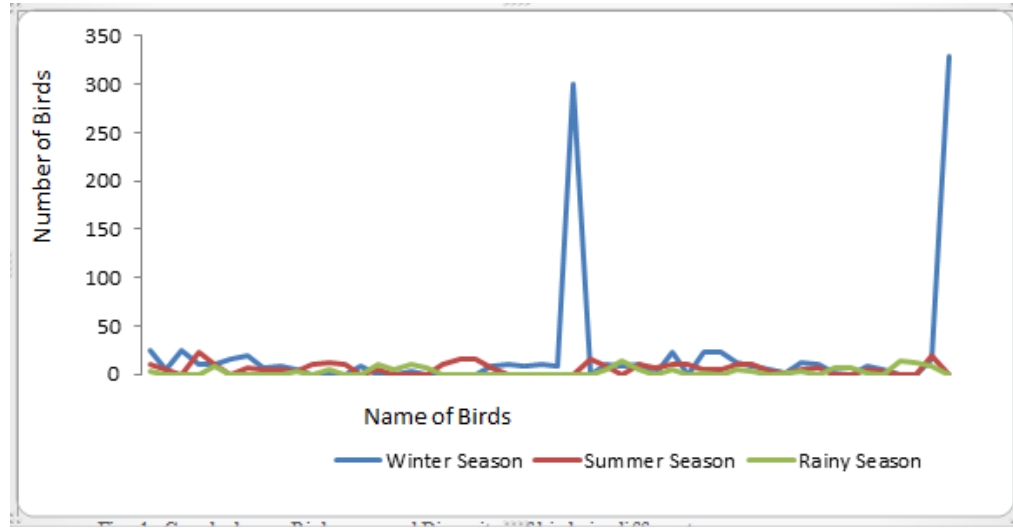

Fig. 1. Graph shows Richness and Diversity of birds in different season.

I observed 97 species of bird visited the agricultural area. Out of that 28 species are wetland habitat, 19 species are forest habitat and 50 species are agricultural habitat. Birds of agricultural area are granivores, frugivores, insectivores and omnivores (Shindsa and Saini 1994, Sekercioglu 2012). Seed- dispersing frugivores ,granivores, omnivores and pollinating nectarivores are higher in agricultural area(Toor et al., 1986). The Simpson Diversity index of birds of agricultural habitat in winter season is 0.35 , in summer season is 0.1 and in rainy season is 0.05 , it indicate that birds richness and diversity in winter season as compared to summer and rainy season. I also observed that considerable difference in functional distribution, specialization and population size among the agricultural bird communities (Sekercioglu 2010). The population of Starling (Sturnus roseus) and Baya Weaver (Ploceus philippinus) are more in winter season. Seasonal change in population density and other indices of few species in agricultural area (Toor et al., 1986). The wetland and forest habitat of birds are occasionally or regularly visit agricultural area in three seasons it may be due to replacement of forests with agricultural area and decrease wetlands (Waltert et al.,2005, Peh et al., 2006, Leyequie et al.,2010,Sekercioglu 2010).

\section{References}

[1]. Ali S(1971). Sunder Lal Hora memoral lecture.Ornithology in India; its past, present and future; pro.Indian Natl.Sci.Acad.B 37,99-113.

[2]. $\quad$ Ali S (1996) .The Book of Indian Birds. Bom.Nat.His.Soc.Oxford Uni.Press 1-354

[3]. Abdar (2007). Ph.D.Thesis. Dr.Babasahab Ambedkar Marathwada University, Aurangabad.

[4]. Bibby CJ, Burgess ND and Hill DA (1992).Bird census Techniques Academic press, London.67-84.

[5]. Bikram Grewal (2000). Birds of the Indian Subcontinent, published by Local Colour Limited Quary Bay, Hong Kong, 1-213.

[6]. Clough Y, Barkmann J (2011). Combining high biodiversity with high yield in tropical agroforests. Proc. Natl. Acad.Sci. USA, $108,8311-8316$.

[7]. Grimmett R,Carol I, Inskipp T (2010). Birds of Indian Subcontinent. Oxford Pocket Guide, Published by Christopher Helm, London, 1-383.

[8]. Kazmierczak K (2009). Om Field Guides Birds of India Sri Lanka, Pakistan, Nepal, Bhutan, Bangladesh and Maldives. Published by OM Books international,Uttar Pradesh, India,1-352.

[9]. Leyequien E, de Boer WF, Toledo VM (2010). Bird community composition in a shaded coffee agro-ecological matrix in Puebia, Mexico; the effect of landscape heterogeneity at multiple spatial scale. Biotropica 42,236-245.

[10]. Naidoo R(2004) Species richness and community of songbirds in a tropical forest-agricultural landscape. Anim. Conserv.7, 93105.Connor R, Shrubb M (1986) Farming and Birds Cambridge; Cambridge Uni. Press.

[11]. Peh KSH, Sodhi NS, de Jong J, Sekercioglu CH, Yap CAM, Lim SLH (2006)

[12]. Conservation value of degraded habitats and forest birds in southern Peninsular Malaysia. Divers Distrib 12, 572-581.

[13]. Perfecto I, Vandermeer J (2008) Biodiversity conservation in tropical agro-ecosystems - a new conservation paradigm. Ann NY Acad Scip 173-200.

[14]. Shindsa and Saini (1994) Agricultural Ornithology: an Indian Perspective. J.Biosci.19(4), 391-402.

[15]. Sekercioglu CH (2010) Bird functional diversity and ecosystem services in tropical forest, agroforests and agricultural area. J.Ornithol, S153-161.

[16]. Toor HS, Kaur H, Dhindsa MS (1986) Community structure and feeding ecology of birds at a grain store in Punjab(India), Trop.Set.26,233-247.

[17]. Tscharntke T, Sekercioglu CH, Dietsch TV, Sodhi NS, Hoehn P, Tylianakis JM (2008) Landscape constraints on functional diversity of birds and Insect in tropical agro-ecosystems . Ecology 89,944-951.

[18]. Van Bael SA, Philpott SM, Greenberg R, Bichier P, Barber NA, Mooney KA, Gruner DS (2008) Birds as predators in tropical agroforestry system,.Ecology 89,928-934.

[19]. Waltert M, Bobo KS, Sainge NH, Fermon H, Muhlenberg M (2005) From forest to farmland; habitat effect on Afrotropical forest bird diversity. Ecol. Appl. 15,1351-1366.

[20]. Wang ZJ, Young SS (2003) Differences in bird diversity between two swidden agricultural sites in mountainous terrain, Xishuangbanna, Yunnan, China. Bio.Conserv 110, 231-243. 